\title{
Study on Combined Determination of Serum NT-proBNP and H-FABP Levels for Diagnosis of Acute Heart Failure
}

\author{
Yanmei Guo, Yongwei Li, Yulin Yang, Yanqing Ma
}

Henan Province Hospital of Traditional Chinese Medicine, Zhengzhou, Henan, 450002

Keywords: NT-proBNP; H-FABP; AHF; diagnostic value

\begin{abstract}
To investigate the diagnostic value of serum N-terminal pro-brain natriuretic peptide (NT-proBNP) and heart-type fatty acid-binding protein (H-FABP) levels in patients with acute heart failure (AHF), a total of 96 patients with suspected AHF in our hospital from March 2016 to February 2018 were selected as subjects. Electrocardiogram, echocardiography and other imaging examinations and clinical features were used as "gold standard". Serum NT-proBNP and H were detected. -FABP levels were analyzed for their individual detection and combined results, and the value of separate detection and combined detection in the diagnosis of AHF was compared. RESULTS: Of the 96 suspected AHF patients selected in this study, 67 were diagnosed by electrocardiographic and echocardiographic imaging and clinical features. 54 were detected by serum NT-proBNP and 52 were detected by serum H-FABP. 65 cases were detected by combined detection of serum NT-proBNP and H-FABP. The combined detection sensitivity, accuracy, and negative predictive value of serum NT-proBNP and H-FABP were higher than that of serum NT-proBNP and H-FABP alone $(\mathrm{P}<0.05)$; the combined detection specificity, positive predictive value, and single detection contrast were not significant. Difference $(\mathrm{P}>0.05)$. The combined detection of serum NT-proBNP and H-FABP in early stage of AHF patients has higher sensitivity and accuracy in the diagnosis of diseases, and can provide data support for early clinical prevention and treatment of cardiovascular events, and this method has a high negative predictive value.
\end{abstract}

\section{Introduction}

Acute heart failure (Acute heart failure, AHF) is mostly on the basis of the original chronic heart failure sudden onset or acute exacerbation, disability and mortality is high, seriously endangering the lives of patients. Early diagnosis and timely symptomatic treatment of such patients are extremely critical and can effectively prevent the occurrence and development of cardiovascular diseases. The current clinical indicators for the diagnosis of AHF are serum cardiac fatty acid-binding protein (H-FABP) and N-terminal pro-brain natriuretic peptide (NT-proBNP). The former is a small-molecule protein mainly distributed in cardiomyocytes. After injury, the substance will be released into the blood; the latter is a component of the natriuretic peptide family, and its synthesis and release are achieved through the ventricular myocytes, which is one of the most intuitive markers of clinical detection of ventricular function [1-2]. In order to further study the diagnostic value of the above two substances in AHF, 96 patients with suspected AHF in our hospital were selected as subjects to analyze the effect of the combined detection on the diagnostic efficacy. The report is as follows.

\section{Materials and Methods}

General data 96 patients with suspected AHF in our hospital from March 2016 to February 2018 were selected as the study subjects, 52 males and 44 females, aged 48-80 years, mean (67.19 \pm 6.71$)$ years old, body mass index $19 \sim 26 \mathrm{~kg} / \mathrm{m} 2$, mean $(22.64 \pm 2.58) \mathrm{kg} / \mathrm{m} 2$, including 41 patients with coronary heart disease, 26 patients with hypertension, 6 patients with dilated cardiomyopathy, and 7 patients with valvular heart disease . This study was approved by the Medical Ethics Committee of our hospital.

Inclusion and Exclusion Criteria (1) Inclusion Criteria: All are accompanied by different degrees 
of exertional dyspnea, orthopnea, suffocation, paroxysmal dyspnea and other symptoms and signs; all by ECG, echocardiography and other imaging examinations The onset time was < 3h; all informed and signed the consent form. (2) Exclusion criteria: those with severe consciousness abnormalities; those with unstable angina; those with severe lung disease and myocardial infarction; those with severe liver and kidney disease; those with psychosis.

Methods Five $\mathrm{ml}$ of venous abdominal venous blood were collected after admission and centrifuged for 5 minutes (3000 r/min). The supernatant was stored at $-75^{\circ} \mathrm{C}$ for testing. The serum H-FABP level was detected by colloidal gold immunochromatographic assay. The instrument used was PUZS-300X automatic biochemical analyzer from Beijing Pulang New Technology Co., Ltd., which was supplied by the reagent manufacturer. The serum NT was detected by chemiluminescent microparticle immunoassay. -proBNP level, the equipment used is the United States Beckman Coulter company UniCel DxI 800 type, the reagents used are provided by the manufacturer. All are strictly operated by the same specialist laboratory technicians with reference to the kit instructions. Positive diagnostic criteria: serum H-FABP levels $>60 \mathrm{ng} / \mathrm{ml}$; serum NT-proBNP levels> $125 \mathrm{pg} / \mathrm{ml}$.

Observation indicators (1) Analysis of serum H-FABP, NT-proBNP alone and combined detection results. (2) The sensitivity, specificity, accuracy, positive predictive value and negative predictive value of H-FABP and NT-proBNP were tested separately.

Statistical analysis Data were processed by SPSS 21.0. Count data (sensitivity, accuracy, negative predictive value, specificity, and positive predictive value) were expressed in $\mathrm{n}(\%)$. The $\chi^{2}$ test was performed. $\mathrm{P}<0.05$ was considered statistically significant. .

\section{Results}

Four-cell table This study selected 96 suspected AHF patients, confirmed by ECG, echocardiography and other clinical features of 67 cases, using serum NT-proBNP detection of 54 cases, serum H-FABP detection In 52 cases, 65 cases were detected by combined detection of serum NT-proBNP and H-FABP, as shown in Table 1.

Table 1 Four-square table ( $\mathrm{n}=96)$

\begin{tabular}{llllllll}
\hline \multirow{2}{*}{$\begin{array}{l}\text { Clinically } \\
\text { confirmed }\end{array}$} & NT-proBNP & + & H-FABP & \multicolumn{3}{l}{ Joint detection } & \\
\cline { 2 - 7 } & & - & + & - & + & - & Total \\
\hline+ & 54 & 13 & 52 & 15 & 65 & 2 & 67 \\
- & 2 & 27 & 1 & 28 & 3 & 26 & 29 \\
Total & 56 & 40 & 53 & 43 & 68 & 28 & 96 \\
\hline
\end{tabular}

Test results Serum NT-proBNP, H-FABP combined detection sensitivity, accuracy, negative predictive value higher than the serum NT-proBNP, H-FABP alone detection $(\mathrm{P}<0.05)$; combined detection of specificity, positive predictive value and detection alone There was no significant difference between the controls ( $\mathrm{P}>0.05)$. See Table 2 .

Table 2 Test result n (\%)

\begin{tabular}{llllll}
\hline Items & Sensitivity & Specificity & Accuracy & $\begin{array}{l}\text { Negative } \\
\text { predictive value }\end{array}$ & $\begin{array}{l}\text { Positive } \\
\text { predictive value }\end{array}$ \\
\hline NT-proBNP & $80.60 \%(54 / 67)$ & $93.10 \%(27 / 29)$ & $84.38 \%(81 / 96)$ & $67.50 \%(27 / 40)$ & $96.43 \%(54 / 56)$ \\
H-FABP & $77.61 \%(52 / 67)$ & $96.55 \%(28 / 29)$ & $83.33 \%(80 / 96)$ & $65.12 \%(28 / 43)$ & $98.11 \%(52 / 53)$ \\
Joint & $97.01 \%(65 / 67)$ & $89.66 \%(26 / 29)$ & $94.79 \%(91 / 96)$ & $92.86 \%(26 / 28)$ & $95.59 \%(65 / 68)$ \\
detection & & & & & \\
\hline$\chi^{2}$ & 11.519 & 1.074 & 7.048 & 7.567 & 0.588 \\
$P$ & 0.003 & 0.585 & 0.030 & 0.023 & 0.745 \\
\hline
\end{tabular}




\section{Discussion}

Heart failure (HF) is the end-stage manifestation of many cardiovascular diseases, and is caused by overwork and abnormal blood discharge. The clinical manifestations of edema, dyspnea, wheezing, etc., if not timely treatment will burst AHF, life-threatening. Therefore, patients with AHF need to be diagnosed early and given targeted treatment measures to prevent cardiovascular events.

In the past, the "gold standard" for clinical diagnosis of AHF was the joint detection of ECG, echocardiography, and other imaging methods, but its operation was relatively complicated and costly, and it was not suitable for clinical screening. Most patients were already accompanied by AHF symptoms when they visited the hospital. Affect the treatment effect and prognosis [3]. In recent years, with the continuous development and innovation of clinical laboratory medicine and biology, it has provided a new direction for early clinical screening of high-risk AHF populations. Serum NT-proBNP and H-FABP are clinically used clinically for the diagnosis of cardiovascular diseases in recent years. Among them, H-FABP is often used in the assessment of acute myocardial infarction, acute myocardial injury area, and severity of AHF. Clinical studies have confirmed that serum H-FABP is highly sensitive and specific in patients presenting with chest pain and can provide data support for the early and reasonable treatment of such patients. It can reduce misdiagnosis in patients with non-cardiac causes of chest pain. Helps with prompt treatment and restores their physical function quickly [4-6]. In addition, NT-proBNP is synthesized and released by ventricular myocytes and has a higher degree of specificity. NT-proBNP has many advantages such as long half-life, excellent in vitro stability, and not easily affected by receptors. It is widely used in clinical ventricular function. Abnormal diagnosis is one of the most intuitive and sensitive and specific indicators in the early stages of these diseases. Clinical reports indicate that when recombinant human B-type natriuretic peptides produced by gene recombination technology are used in clinical treatment, they will not interfere with the results of serum NT-proBNP detection [7]. Researchers Li Fen-xia et al. [8] pointed out that serum levels of NT-proBNP in patients with AHF are significantly elevated and have a positive correlation with the severity of the disease.

Based on the above results, in this study, serum NT-proBNP and H-FABP levels were detected in 96 suspected AHF patients. 54 cases were detected by serum NT-proBNP detection, and 52 cases were detected by serum H-FABP detection. Serum NT-proBNP Sixty-five cases were detected by H-FABP combined detection, and the combined detection sensitivity was $97.01 \%$, which was higher than that of the single detection $(\mathrm{P}<0.05)$, indicating that this scheme is feasible in early diagnosis of AHF patients. In addition, some scholars pointed out that the urinary and edema symptoms of kidney disease, abdominal pain and abdominal distention and other symptoms of gastroenteritis, liver cirrhosis with edema of the lower extremities should be identified with AHF, these symptoms are similar to AHF, if misdiagnosis Cause medical accidents and delay the timing of patients' treatment [9]. The analysis of this situation in this study found that the combined detection accuracy and negative predictive value of serum NT-proBNP and H-FABP was higher than that of the single test $(\mathrm{P}<0.05)$, which helps to reduce the incidence of missed diagnosis and can be used for serum NT-proBNP. The abnormal H-FABP patients were further tested by imaging to confirm the diagnosis and provide data support for targeted treatment.

In summary, early detection of serum NT-proBNP and H-FABP levels in patients with AHF has a high sensitivity and accuracy in the diagnosis of disease, and can provide data support for early clinical prevention and treatment of cardiovascular events, and this method negative predictive value High energy can avoid missed diagnosis.

\section{References}

[1] Liu N, Liu M, Wang ZJ, et al. Effects of Danshen Dripping Pills and ARB on serum NT-proBNP, $\mathrm{H}-\mathrm{FABP}$ and Nexilin in elderly patients with chronic heart failure[J]. Chinese Archives of Traditional Chinese Medicine,2016,34 (1): 251-254. 
[2] Liu Li,Han Yubo,Zou Guoliang,et al.Relationship between TCM syndrome type and free fatty acid and cardiac fatty acid binding protein in patients with heart failure with preserved ejection fraction[J].Liaoning Journal of Traditional Chinese Medicine,2016,43(12) ): 2555-2558.

[3] Jiang Xiaoxia, Long Wenjun, Zhang Yi. Clinical investigation of left ventricular function, heart rate variability, time domain, electrocardiogram, and other indicators for the diagnosis of heart failure using echocardiography and electrocardiography [J]. Imaging Research and Medical Applications, 2017, 1(10): 44-46.

[4] Wang Xiaole, Yu Zhouhai, Wang Yizhen, et al. Early diagnosis of non-ST-elevation acute coronary syndrome with combined detection of IMA, H-FABP, and cTnI [J]. Marked Immunoassay and Clinical, 2016,23 (4): 357-359.

[5] Pan Caiying, Li Jingchun, Long Zhenhong, et al. Specificity, sensitivity and correlation of two myocardial markers and two myocardial enzymes in the diagnosis and therapeutic judgment of acute myocardial infarction[J]. Laboratory Medicine and Clinic, 2016,13(1):185-188.

[6] Liu Yingli, Tang Liangqiu, Fan Wenmao, et al. Correlation between serum cardiac fatty acid-binding protein levels and prognosis of patients with acute ST-segment elevation myocardial infarction before and after direct percutaneous coronary intervention [J]. Journal of interventional cardiology, 2016, 24(8): 433-438.

[7] Zhang Yuanyuan, Li Xiaodong. Effect of freeze-dried recombinant human brain natriuretic peptide in continuous low-dose pumping for $72 \mathrm{~h}$ on plasma levels of NT-proBNP and LVEF in patients with chronic heart failure[J]. Practical Pharmacy and Clinic,2016,19(6): 677-680.

[8] Li Fanxia, Chen Fenghui, Han Gang, et al. Effects of Zuoxie Mengdan combined with Naoxintong Capsule on acute heart failure and its effects on serum levels of NT-proBNP, Galectin-3, ET-1, and CystatinC [J]. Advances in modern biomedicine, 2017, 17(25):4888-4891.

[9] Jin Bing, Zhu Shuyang, Chen Bi, et al. The value of pulse oscillation in differentiating chronic obstructive pulmonary disease from acute exacerbations and chronic heart failure caused by chronic heart failure[J]. Jiangsu Medical Science, 2014, 40(15) : 1788-1790. 